\title{
Nonequilibrium quantum dynamics in the condensed phase via the generalized quantum master equation
}

\author{
Ming-Liang Zhang, Being J. Ka, and Eitan Geva ${ }^{\text {a) }}$ \\ Department of Chemistry and FOCUS Center, University of Michigan, Ann Arbor, Michigan 48109-1055
}

(Received 5 May 2006; accepted 5 June 2006; published online 28 July 2006)

\begin{abstract}
The Nakajima-Zwanzig generalized quantum master equation provides a general, and formally exact, prescription for simulating the reduced dynamics of a quantum system coupled to a quantum bath. In this equation, the memory kernel accounts for the influence of the bath on the system's dynamics, and the inhomogeneous term accounts for initial system-bath correlations. In this paper, we propose a new approach for calculating the memory kernel and inhomogeneous term for arbitrary initial state and system-bath coupling. The memory kernel and inhomogeneous term are obtained by numerically solving a single inhomogeneous Volterra equation of the second kind for each. The new approach can accommodate a very wide range of projection operators, and requires projection-free two-time correlation functions as input. An application to the case of a two-state system with diagonal coupling to an arbitrary bath is described in detail. Finally, the utility and self-consistency of the formalism are demonstrated by an explicit calculation on a spin-boson model. (C) 2006 American Institute of Physics. [DOI: 10.1063/1.2218342]
\end{abstract}

\section{INTRODUCTION}

A common approach for studying quantum dynamics in the condensed phase is based on the observation that, in practice, one can often directly probe and/or manipulate only a small number of degrees of freedom (DOFs). The subsystem subject to direct observation and/or manipulation may correspond to the reaction coordinate, a relaxing vibrational mode of a solute molecule, or an optically active transition in a solvated molecule. This observation naturally leads to a strategy that combines an accurate description of the subsystem, which will be referred to as the system from now on, with a minimal, yet accurate treatment of the rest of the DOF, which will be referred to as the bath. The key to the success of such an approach relies on one's ability to accurately filter out those aspects of the many-body bath dynamics which affect the system.

A general approach for dealing with the nonequilibrium quantum dynamics of a system coupled to a bath can be based on the Nakajima-Zwanzig generalized quantum master equation (GQME) ${ }^{1-16}$ This GQME can be derived with the help of projection operator techniques. It requires as input such quantities as the memory kernel, which accounts for bath-induced non-Markovian dissipative dynamics, and the inhomogeneous term, which reflects initial system-bath correlations. The calculation of these quantities is associated with two major complications: (1) they involve the manybody dynamics of the overall system (i.e., system + bath) and (2) they depend on the projection operator in a nontrivial manner (i.e., exponentially). A very popular approach for bypassing at least the second difficulty is based on employing the following approximations: (1) Calculating the memory kernel at the limit of weak system-bath coupling,

${ }^{\text {a)} E l e c t r o n i c ~ m a i l: ~ e i t a n @ u m i c h . e d u ~}$
(2) assuming that the bath fluctuates on a time scale which is much faster than the system's relaxation rate, and (3) neglecting the inhomogeneous term. These assumptions lead to a Markovian quantum master equation of the Bloch-Redfield type. The corresponding memory kernel is projection independent and can be given in terms of free-bath two-time correlation functions. ${ }^{6,8,10-12,14,15,17-35}$ Unfortunately, the assumptions regarding weak system-bath coupling and Markovity also make it impossible to account for such important phenomena as solvation dynamics and solvent memory effects, which are central to solution chemistry. Thus, the development of new ways for using the GQME beyond the limit of weak system-bath coupling and Markovity is clearly highly desirable.

In a previous paper, ${ }^{16}$ we presented a theoretical framework for calculating the Nakajima-Zwanzig memory kernel for arbitrary system-bath coupling. The proposed scheme was based on a set of two, formally exact, coupled inhomogeneous Volterra equations of the second kind, which allowed for the calculation of the projection-dependent memory kernel from projection-free input that could be put in terms of overall system two-time correlation functions. Since the majority of DOF usually belong to the bath, the computational cost of calculating overall system two-time correlation functions is comparable to that of calculating free-bath ones. Thus, the computational cost of calculating the memory kernel for arbitrary system-bath coupling within the new approach is comparable to that of calculating it in the limit of weak system-bath coupling.

A demonstration of the new approach in the case of a two-level system linearly coupled to a harmonic bath has been provided in Ref. 16. In this case, it was possible to compute the input two-time correlation functions in a numerically exact manner, via the quasiadiabatic propagator path integral (QUAPI) method. ${ }^{36,37}$ A similar numerically ex- 
act calculation of two-time quantum-mechanical correlation functions in the case of many-body anharmonic systems is not feasible. However, a variety of approximate semiclassical and mixed quantum-classical techniques for computing these quantities are available. ${ }^{38}$ Indeed, in previous papers we have demonstrated the applicability of the linearized semiclassical $^{39}$ and mixed quantum-classical Liouville ${ }^{40}$ methods for computing the memory kernel beyond the limit of weak system-bath coupling.

In the present paper, we propose a new GQME-based approach to nonequilibrium quantum dynamics in the condensed phase. The new approach represents a significant improvement over the scheme proposed in Ref. 16 in the following respects:

(1) Whereas the approach in Ref. 16 was derived for a specific choice of projection operator, the new approach can accommodate a wide range of possible projection operators.

(2) The calculation of the memory kernel within the new approach is based on solving a single inhomogeneous Volterra equation of the second kind, instead of the two coupled Volterra equations required by the approach in Ref. 16.

(3) The new approach includes a new scheme for computing the inhomogeneous term, which requires projection-free input similar to that used for calculating the memory kernel.

(4) The new approach is applicable to driven systems with explicitly time-dependent Hamiltonians.

Another goal of this paper is to consider the application of the new approach to the case of a two-state system with diagonal system-bath coupling. This application further illustrates some of the unique features of the new approach, and sets the stage for its future application to the calculation of optical spectra in condensed phase systems.

The structure of the remainder of this paper is as follows. A short overview of the GQME formalism is provided in Sec. II. The new theoretical framework for calculating the memory kernel and inhomogeneous term, in the case of arbitrary system-bath coupling and initial state of the overall system, is outlined in Sec. III. The new framework is applied to the case of a two-state system diagonally coupled to a bath in Sec. IV. An illustrative application to a spin-boson model is described in Sec. V. We end with a summary of the main results in Sec. VI.

\section{PRELIMINARY CONSIDERATIONS}

Consider an overall system with the following general quantum-mechanical Hamiltonian:

$$
\hat{H}=\hat{H}_{s}+\hat{H}_{b}+\hat{H}_{b s} \text {. }
$$

Here, $\hat{H}_{s}$ is the system Hamiltonian, $\hat{H}_{b}$ is the bath Hamiltonian, and $\hat{H}_{b s}$ is the coupling between them (throughout this paper, capped symbols such as $\hat{H}$ represent operators). In this and the following sections, we will assume that $\hat{H}_{s}$ is time independent. An extension of the formalism to the case of a time-dependent system Hamiltonian is described in Appendix A.

We assume, without loss of generality, that

$$
\left\langle\hat{H}_{b s}\right\rangle_{R} \equiv \operatorname{Tr}_{b}\left(\hat{H}_{b s} \hat{R}\right)=0 .
$$

Here, $\operatorname{Tr}_{b}$ corresponds to a partial trace over the Hilbert space of the bath, and $\hat{R}$ is an overall system operator which must satisfy

$$
\operatorname{Tr}_{b}(\hat{R})=\hat{1}_{s}
$$

where $\hat{1}_{s}$ is the unity operator in the system's Hilbert space. The operator $\hat{R}$ will be used later as part of the projection operator [cf. Eq. (11)].

It should be noted that one can always redefine the system Hamiltonian and system-bath coupling term such that the condition in Eq. (2) is satisfied. More specifically, suppose that

$$
\hat{H}=\hat{H}_{s}^{\prime}+\hat{H}_{b}+\hat{H}_{b s}^{\prime},
$$

such that $\operatorname{Tr}_{b}\left(\hat{H}_{b s}^{\prime} \hat{R}\right) \neq 0$. Then, one may define $\hat{H}_{s}=\hat{H}_{s}^{\prime}$ $+\left\langle\hat{H}_{b s}^{\prime}\right\rangle_{R}$ and $\hat{H}_{b s}=\hat{H}_{b s}^{\prime}-\left\langle\hat{H}_{b s}^{\prime}\right\rangle_{R}$, such that $\left\langle\hat{H}_{b s}\right\rangle_{R}=0$. It should also be noted that, at least formally, $\hat{H}_{b s}$ can always be put in the form

$$
\hat{H}_{b s}=\sum_{j} \hat{B}_{j} \otimes \hat{S}_{j},
$$

where $\hat{B}_{j}$ and $\hat{S}_{j}$ are independent bath and system operators, respectively. $\left\langle\hat{H}_{b s}\right\rangle_{R}=0$ implies that $\left\langle\hat{B}_{j}\right\rangle_{R}=0$ for all $\left\{\hat{B}_{j}\right\}$.

The state of the overall system at time $t$ is described by the density operator $\hat{\rho}(t)$, whose dynamics is dictated by the Liouville equation as follows:

$$
\begin{aligned}
\frac{d}{d t} \hat{\rho}(t) & =-\frac{i}{\hbar}[\hat{H}, \hat{\rho}(t)] \equiv-\frac{i}{\hbar} \mathcal{L} \hat{\rho}(t) \\
& =-\frac{i}{\hbar}\left[\hat{H}_{s}+\hat{H}_{b}+\hat{H}_{b s}, \hat{\rho}(t)\right] \\
& \equiv-\frac{i}{\hbar}\left(\mathcal{L}_{s}+\mathcal{L}_{b}+\mathcal{L}_{b s}\right) \hat{\rho}(t) .
\end{aligned}
$$

The state of the system at time $t$ is described by the reduced density operator

$$
\hat{\sigma}(t)=\operatorname{Tr}_{b}[\hat{\rho}(t)] \text {. }
$$

Unlike $\hat{\rho}(t)$, the time evolution of $\hat{\sigma}(t)$ is not unitary, and therefore cannot be described by a Liouville-type equation of motion. Several formal approaches for describing such nonunitary dynamics have been proposed in the past. ${ }^{1-9}$ The approach that we will adopt in this paper is based on projection operator techniques and leads to a formally exact nonMarkovian equation of motion for $\hat{\sigma}(t)$, which is known as the Nakajima-Zwanzig GQME. ${ }^{1-12,14-16}$ An outline of the derivation of the GQME is given below for the case of a time-independent system Hamiltonian (the generalization to the case of a time-dependent system Hamiltonian is described in Appendix A).

Consider a linear projection operator $\mathcal{P}$, which satisfies 


$$
\mathcal{P}^{2}=\mathcal{P} .
$$

The specific choice of $\mathcal{P}$ is dictated by what we choose to project out, as well as convenience (see below). Following the standard procedure, one operates on Eq. (6) from the left with either $\mathcal{P}$ or its complimentary projection operator

$$
\mathcal{Q}=\hat{1}-\mathcal{P},
$$

where $\hat{1}=\hat{1}_{s} \otimes \hat{1}_{b}$ is the overall system unity operator. This results in a set of two coupled linear first order differential equations, one for $\mathcal{P} \hat{\rho}(t)$ and another for $\mathcal{Q} \hat{\rho}(t)$. Explicitly solving the equation for $\mathcal{Q} \hat{\rho}(t)$ and substituting the result in the equation for $\mathcal{P} \hat{\rho}(t)$ then leads to the GQME in its most general form as follows:

$$
\begin{aligned}
\frac{d}{d t} \mathcal{P} \hat{\rho}(t)= & -\frac{i}{\hbar} \mathcal{P} \mathcal{L} \mathcal{P} \hat{\rho}(t)-\frac{1}{\hbar^{2}} \int_{0}^{t} d \tau \mathcal{P} \mathcal{L} e^{-i \mathcal{Q} L t / \hbar} \mathcal{Q} \mathcal{L} \mathcal{P}(t-\tau) \\
& -\frac{i}{\hbar} \mathcal{P} \mathcal{L} e^{-i \mathcal{Q} L t / \hbar} \mathcal{Q} \hat{\rho}(0) .
\end{aligned}
$$

It should be noted that in deriving Eq. (10), we have assumed that $\hat{H}_{s}$ is time independent.

The choice of $\mathcal{P}$ is dictated by the desire to project out the bath DOF, and is given by

$$
\mathcal{P}(\cdot)=\hat{R} \operatorname{Tr}_{b}(\cdot),
$$

where $\hat{R}$ is as in Eq. (3). Substituting Eq. (11) in Eq. (10), then leads to the following GQME for $\hat{\sigma}(t)$ :

$$
\begin{aligned}
\frac{d}{d t} \hat{\sigma}(t)= & -\frac{i}{\hbar} \mathcal{L}_{s} \hat{\sigma}(t)-\frac{i}{\hbar} \operatorname{Tr}_{b}\left[\mathcal{L}_{b s} \hat{R} \hat{\sigma}(t)\right] \\
& -\int_{0}^{t} d \tau \mathcal{K}(\tau) \hat{\sigma}(t-\tau)+\hat{I}(t) .
\end{aligned}
$$

Here, $\mathcal{K}(\tau)$ is the memory kernel (a system superoperator) and $\hat{I}(t)$ is the inhomogeneous term (a system operator). They are explicitly given by

$$
\begin{aligned}
& \mathcal{K}(\tau)=\frac{1}{\hbar^{2}} \operatorname{Tr}_{b}\left\{\mathcal{L}_{b s} e^{-i \mathcal{Q} \mathcal{L} \tau / \hbar} \mathcal{Q} \mathcal{L} \hat{R}\right\}, \\
& \hat{I}(t)=-\frac{i}{\hbar} \operatorname{Tr}_{b}\left\{\mathcal{L}_{b s} e^{-i \mathcal{Q} \mathcal{L} t / \hbar} \mathcal{Q} \hat{\rho}(0)\right\} .
\end{aligned}
$$

In deriving Eqs. (12)-(14), we have used the following identities:

$$
\begin{aligned}
& \operatorname{Tr}_{b}\left(\mathcal{L}_{b} \cdot\right)=0, \quad \operatorname{Tr}_{b}\left(\mathcal{P} \mathcal{L}_{s} \cdot\right)=\operatorname{Tr}_{b}\left(\mathcal{L}_{s} \mathcal{P} \cdot\right)=\mathcal{L}_{s} \operatorname{Tr}_{b}(\cdot), \\
& \operatorname{Tr}_{b}\left(e^{-i \mathcal{Q} L / \hbar} \mathcal{Q} \cdot\right)=0 .
\end{aligned}
$$

The first term on the right-hand side of Eq. (12), $-i \mathcal{L}_{s} \hat{\sigma}(t) / \hbar$, accounts for the unitary bath-free system dynamics. The second term, $-i \operatorname{Tr}_{b}\left[\mathcal{L}_{b s} \hat{R} \hat{\sigma}(t)\right] / \hbar$, accounts for the bath-induced shift of the system's unitary dynamics. This term vanishes as a result of our assumption that $\left\langle\hat{H}_{b s}\right\rangle_{R}=0$ [cf. Eqs. (2) and (5)]:

$$
\begin{aligned}
\operatorname{Tr}_{b}\left[\mathcal{L}_{b s} \hat{R} \hat{\sigma}(t)\right] & =\operatorname{Tr}_{b}\left[\hat{H}_{b s} \hat{R} \hat{\sigma}(t)\right]-\operatorname{Tr}_{b}\left[\hat{R} \hat{\sigma}(t) \hat{H}_{b s}\right] \\
& =\sum_{j} \operatorname{Tr}_{b}\left[\hat{B}_{j} \otimes \hat{S}_{j} \hat{R} \hat{\sigma}(t)\right]-\operatorname{Tr}_{b}\left[\hat{R} \hat{\sigma}(t) \hat{B}_{j} \otimes \hat{S}_{j}\right] \\
& =\sum_{j}\left[\hat{S}_{j}\left\langle\hat{B}_{j}\right\rangle_{R} \hat{\sigma}(t)-\left\langle\hat{B}_{j}\right\rangle_{R} \hat{\sigma}(t) \hat{S}_{j}\right]=0
\end{aligned}
$$

The third term, $-\int_{0}^{t} d \tau \mathcal{K}(\tau) \hat{\sigma}(t-\tau)$, represents a nonunitary and non-Markovian bath-induced contribution to the system's dynamics. The fourth term, $\hat{I}(t)$, accounts for projected-out correlations between the system and the bath at $t=0$. It should be noted that the latter two bath-induced terms need not be Hermitian. However, their sum must be Hermitian since $d \hat{\sigma} / d t$ is. We also note that it is possible (although not necessary!) to express the GQME in terms of Hermitian bath-induced terms, for example, by employing a symmetrized projection operator of the form $\mathcal{P}(\cdot)=\left\{\hat{R} \operatorname{Tr}_{b}(\cdot)\right.$ $\left.+\operatorname{Tr}_{b}(\cdot) \hat{R}^{\dagger}\right\} / 2$ (cf. Appendix B).

Equation (12) corresponds to a family of possible equations of motion for a system coupled to a bath. These equations differ with respect to the choice of reference operator, $\hat{R}$, but are otherwise exact and equivalent. It should also be noted that Eq. (12) can accommodate system-bath coupling of arbitrary strength, non-Markovian dynamics, and initial system-bath correlations. The same features are not accommodated by the commonly used Bloch-Redfield quantum master equation. The latter corresponds to an approximate version of Eq. (12), which can be obtained by imposing weak system-bath coupling (in the sense of first order timedependent perturbation theory), Markovity, and the assumption of an uncorrelated initial state.

\section{EVALUATION OF THE MEMORY KERNEL AND INHOMOGENEOUS TERM}

In order to use Eq. (12) in practice, it is necessary to be able to compute $\mathcal{K}(\tau)$ [Eq. (13)] and $\hat{I}(t)$ [Eq. (14)]. A major difficulty in calculating these quantities has to do with their explicit and nontrivial dependence on the projection operator $\mathcal{Q}$. In this section, we propose a general approach that makes it possible to compute $\mathcal{K}(\tau)$ and $\hat{I}(t)$ from projection-free input.

The evaluation of $\mathcal{K}(\tau)$ and $\hat{I}(t)$ is facilitated by the following general identity: ${ }^{41-43}$

$$
e^{-i \mathcal{Q} \mathcal{L} t / \hbar}=e^{-i \mathcal{L} t / \hbar}+\frac{i}{\hbar} \int_{0}^{t} d \tau e^{-i \mathcal{L}(t-\tau) / \hbar} \mathcal{P} \mathcal{L} e^{-i \mathcal{Q} \mathcal{L} \tau / \hbar} .
$$

Equation (17) can be derived by integrating both sides of the differential equation $d\left[e^{i \mathcal{L} \tau / \hbar} e^{-i \mathcal{Q L} \tau / \hbar}\right] / d \tau$ $=(i / \hbar) e^{i \mathcal{L} \tau / \hbar} \mathcal{P} \mathcal{L} e^{-i \mathcal{Q} \mathcal{L} / \hbar}$ from 0 to $t$, and operating on the result from the left by $e^{-i \mathcal{L} t / \hbar}$. This identity has been employed in the past mostly in terms of its Fourier-Laplace transform representation, ${ }^{1,44-48}$ 


$$
\frac{1}{z+i \mathcal{Q L} / \hbar}=\frac{1}{z+i \mathcal{L} / \hbar}+\frac{i}{\hbar} \frac{1}{z+i \mathcal{L} / \hbar} \mathcal{P} \mathcal{L} \frac{1}{z+i \mathcal{Q L} / \hbar}
$$

in a variety of applications of the projection operator formalism. It is extremely general and does not even depend on the actual choices of $\mathcal{P}$ or $\mathcal{L}$.

We first consider the evaluation of the inhomogeneous term, $\hat{I}(t)$. To this end, we substitute Eq. (17) in Eq. (14). Specializing to the projection operator in Eq. (11) then leads to the following operator inhomogeneous Volterra equation of the second kind ${ }^{49}$ for $\hat{I}(t)$ :

$$
\hat{I}(t)=\hat{\Xi}(t)+\Phi(t) \hat{\sigma}(0)+\int_{0}^{t} d \tau \Phi(t-\tau) \hat{I}(\tau) .
$$

Here, $\hat{\Xi}(t)$ and $\Phi(t)$ are projection-free system operator and superoperator, respectively, which are explicitly given by

$$
\hat{\Xi}(t)=-\frac{i}{\hbar} \operatorname{Tr}_{b}\left[\mathcal{L}_{b s} e^{-i \mathcal{L} t / \hbar} \hat{\rho}(0)\right]
$$

and

$$
\Phi(t)=\frac{i}{\hbar} \operatorname{Tr}_{b}\left[\mathcal{L}_{b s} e^{-i \mathcal{L} t / \hbar} \hat{R}\right]
$$

We next consider the evaluation of the memory kernel $\mathcal{K}(\tau)$. To this end, we substitute Eq. (17) in Eq. (13). Specializing once again to the projection operator in Eq. (11) leads to the the following inhomogeneous Volterra equation of the second kind for $\mathcal{K}(\tau)$ :

$$
\mathcal{K}(\tau)=\dot{\Phi}(\tau)+\frac{i}{\hbar} \Phi(\tau) \mathcal{L}_{s}+\int_{0}^{\tau} d \tau^{\prime} \Phi\left(\tau-\tau^{\prime}\right) K\left(\tau^{\prime}\right),
$$

where $\Phi(t)$ is as in Eq. (21).

Equations (19) and (22) constitute compact and general relations between the projection-dependent $\mathcal{K}(\tau)$ and $\hat{I}(t)$ and the projection-free $\Phi(t)$ and $\hat{\Xi}(t)$. Thus, the problem of computing $\mathcal{K}(\tau)$ and $\hat{I}(t)$ translates into that of calculating $\Phi(t)$ and $\hat{\Xi}(t)$, followed by solving Eq. (19) for $\hat{I}(t)$ and Eq. (22) for $\mathcal{K}(\tau)$.

It should be noted that Eqs. (19) and (22) are not unique. More specifically, $\mathcal{K}(\tau)$ and $\hat{I}(t)$ can be related to projectionfree quantities in other ways. For example, an alternative route for computing $\mathcal{K}(\tau)$ can be based on the observation that

$$
\mathcal{K}(\tau)=\dot{\Gamma}(\tau)
$$

where

$$
\Gamma(\tau)=\frac{i}{\hbar} \operatorname{Tr}_{b}\left[\mathcal{L}_{b s} e^{-i \mathcal{Q} \mathcal{L} \tau / \hbar} \hat{R}\right]
$$

Substituting Eq. (17) in Eq. (24) then yields the following equation:

$$
\Gamma(\tau)=\Phi(\tau)+\int_{0}^{\tau} d \tau^{\prime} \Phi\left(\tau-\tau^{\prime}\right)\left(\frac{i}{\hbar} \mathcal{L}_{s}+\Gamma\left(\tau^{\prime}\right)\right)
$$

where $\Phi(\tau)$ is as in Eq. (21). Thus, the projection-dependent $\Gamma(\tau)$ can be obtained from the projection-free $\Phi(\tau)$. Once $\Gamma(\tau)$ is known, $\mathcal{K}(\tau)$ can be easily obtained by taking its time derivative [cf. Eq. (23)]. It can also be verified that Eq. (22) can be obtained from Eq. (25) by taking the time derivative of the latter.

Yet another route for obtaining $\mathcal{K}(\tau)$ from projectionfree input can be derived with the help of the identities

$$
e^{-i \mathcal{Q} \mathcal{L} t / \hbar} \mathcal{Q}=\mathcal{Q} e^{-i \mathcal{L} \mathcal{Q} t / \hbar}
$$

and

$$
e^{-i \mathcal{L} \mathcal{Q} t / \hbar}=e^{-i \mathcal{L} t / \hbar}+\frac{i}{\hbar} \int_{0}^{t} d \tau e^{-i \mathcal{L}(t-\tau) / \hbar} \mathcal{L} \mathcal{P} e^{-i \mathcal{L} \mathcal{Q} \tau / \hbar} .
$$

Upon substituting these identities in Eq. (13), it can be shown that

$$
\mathcal{K}(\tau)=\dot{\Phi}(\tau)+i \int_{0}^{\tau} d \tau^{\prime} \dot{\Phi}\left(\tau-\tau^{\prime}\right) \mathcal{K}^{\prime}\left(\tau^{\prime}\right)
$$

where

$$
\mathcal{K}^{\prime}(\tau)=\frac{1}{\hbar} \operatorname{Tr}_{b}\left(e^{-i \mathcal{L Q} \tau / \hbar} \mathcal{L} \hat{R}\right) .
$$

Substituting the identity in Eq. (27) back in Eq. (29) then leads to the following equation:

$$
\mathcal{K}^{\prime}(\tau)=\Lambda(\tau)+i \int_{0}^{\tau} d \tau^{\prime} \Lambda\left(\tau-\tau^{\prime}\right) \mathcal{K}^{\prime}\left(\tau^{\prime}\right)
$$

where

$$
\Lambda(\tau)=\frac{1}{\hbar} \operatorname{Tr}_{b}\left[e^{-i \mathcal{L} \tau / \hbar} \mathcal{L} \hat{R}\right]
$$

Thus, given the projection-free $\Lambda(\tau)$ as input, Eq. (30) can be solved for the projection-dependent $\mathcal{K}^{\prime}(\tau)$. The latter can then be substituted in Eq. (28), which can be solved for $\mathcal{K}(\tau)$, with the projection-free $\Phi(\tau)$ as input. In the case where $\hat{R}$ $=\hat{\rho}_{b}^{\mathrm{eq}}=e^{-\beta \hat{H}_{b}} / \operatorname{Tr}_{b}\left(e^{-\beta \hat{H}_{b}}\right)$, Eqs. (30) and (28) reduce to Eqs. (9) and (10) of Ref. 16 [to this end, note that $\dot{\Phi}(\tau), \mathcal{K}^{\prime}(\tau)$, and $\Lambda(\tau)$ coincide with $\mathcal{K}_{1}(\tau), \mathcal{K}_{2}(\tau)$, and $\mathcal{K}_{3}(\tau)$ of Ref. 16, respectively]. At the same time, it should also be noted that Eq. (22) provides a more convenient route to $\mathcal{K}(\tau)$ because it involves solving only one Volterra equation and a smaller number of projection-free quantities as input.

The limit of weak system-bath coupling can be obtained by substituting $\hat{R}=\hat{\rho}_{b}^{\mathrm{eq}}=e^{-\beta \hat{H}_{b}} / \operatorname{Tr}_{b}\left(e^{-\beta \hat{H}_{b}}\right)$ and $e^{-i \mathcal{Q L} \tau / \hbar}$ $\approx e^{-i \mathcal{Q}\left(\mathcal{L}_{b}+\mathcal{L}_{s}\right) \tau / \hbar}$ in Eq. (24) as follows: 


$$
\begin{aligned}
\Gamma(\tau) & \approx \frac{i}{\hbar} \operatorname{Tr}_{b} \mathcal{L}_{b s} e^{-i \mathcal{Q}\left(\mathcal{L}_{b}+\mathcal{L}_{s}\right) \tau / \hbar} \hat{\rho}_{b}^{\mathrm{eq}} \\
& =\frac{i}{\hbar} \operatorname{Tr}_{b} \mathcal{L}_{b s}\left(\mathcal{P}+\mathcal{Q} e^{-i\left(\mathcal{L}_{b}+\mathcal{L}_{s}\right) \tau / \hbar}\right) \hat{\rho}_{b}^{\mathrm{eq}} \\
& =\frac{i}{\hbar} \operatorname{Tr}_{b} \mathcal{L}_{b s} e^{-i\left(\mathcal{L}_{b}+\mathcal{L}_{s}\right) \tau / \hbar} \hat{\rho}_{b}^{\mathrm{eq}}=\Phi(\tau) .
\end{aligned}
$$

The second equality in Eq. (32) can be obtained by rewriting $e^{-i \mathcal{Q}\left(\mathcal{L}_{b}+\mathcal{L}_{s}\right) \tau / \hbar}$ in terms of its Taylor expansion and noting that $\mathcal{Q}\left(\mathcal{L}_{b}+\mathcal{L}_{s}\right) \mathcal{Q}=\mathcal{Q}\left(\mathcal{L}_{b}+\mathcal{L}_{s}\right)$. The third equality is based on the fact that $\operatorname{Tr}_{b}\left[\mathcal{L}_{b s} \hat{R}\right]=0$. It should be noted that $\Gamma(\tau)$ coincides with $\Phi(\tau)$ and that $\mathcal{K}(\tau)$ coincides with $\dot{\Phi}(\tau)$ in the limit of weak system-bath coupling.

It is interesting to note that the projection-free input required for calculating $\mathcal{K}(\tau)$ in the case of arbitrary systembath coupling is rather similar to that in the case of weak system-bath coupling. The main difference between the two cases appears to be that the relationship between $\mathcal{K}(\tau)$ and the projection-free input is more direct in the weak coupling case. However, the computational bottleneck in actual applications to anharmonic systems often lies in calculating the projection-free input, rather than in obtaining $\mathcal{K}(\tau)$ from it. Thus, somewhat surprisingly, one finds that the computational cost of calculating $\mathcal{K}(\tau)$ in the case of arbitrary system-bath coupling is expected to be similar to that in the case of weak system-bath coupling.

In order to proceed, it is convenient to put Eqs. (12), (19), (22), and (25) in matrix form. To this end, it is useful to work in terms of the system's $N^{2}$-dimensional Liouville space, where $N$ is the dimension of the system's Hilbert space. Within this formalism, system operators such as $\hat{I}$ are represented by vectors of length $N^{2}$, and superoperators such as $\mathcal{K}(\tau)$ are represented by $N^{2} \times N^{2}$ matrices. The corresponding matrix elements of $\hat{I}(t)$ and $\mathcal{K}(\tau)$ are given by

$$
\begin{aligned}
& I_{i j}(t)=\langle\langle i j \mid \hat{I}(t)\rangle\rangle=\operatorname{Tr}_{s}\left[(|i\rangle\langle j|)^{\dagger} \hat{I}(t)\right]=\langle i|\hat{I}| j\rangle, \\
& \mathcal{K}_{i j, k l}(t)=\langle\langle i j|\mathcal{K}(\tau)| k l\rangle\rangle=\operatorname{Tr}_{s}\left[(|i\rangle\langle j|)^{\dagger} \mathcal{K}(\tau)|k\rangle\langle l|\right] .
\end{aligned}
$$

Here, $\operatorname{Tr}_{s}$ corresponds to tracing over the system's Hilbert space, and $\{|i\rangle,|j\rangle,|k\rangle,|l\rangle\}$ are system states that belong to an orthonormal basis set of one's choice.

The GQME, Eq. (12), can now be put in matrix form as follows:

$$
\begin{aligned}
\frac{d}{d t} \sigma_{i j}(t)= & -\frac{i}{\hbar} \sum_{k, l}\left[\mathcal{L}_{s}\right]_{i j, k l} \sigma_{k l}(t) \\
& -\sum_{k, l} \int_{0}^{t} d \tau \mathcal{K}_{i j, k l}(\tau) \sigma_{k l}(t-\tau)+I_{i j}(t) .
\end{aligned}
$$

Similarly, Eqs. (19), (22), and (25) can also be put in matrix form as follows:

$$
\begin{aligned}
I_{i j}(t)= & \Xi_{i j}(t)+\sum_{k, l} \Phi_{i j, k l}(t) \sigma_{k l}(0) \\
& +\sum_{k, l} \int_{0}^{t} d \tau \Phi_{i j, k l}(t-\tau) I_{k l}(\tau),
\end{aligned}
$$

$$
\begin{aligned}
\mathcal{K}_{i j, k l}(\tau)= & \dot{\Phi}_{i j, k l}(\tau)+\frac{i}{\hbar} \sum_{m, n} \Phi_{i j, m n}(t)\left[\mathcal{L}_{s}\right]_{m n, k l} \\
& +\sum_{m, n} \int_{0}^{\tau} d \tau^{\prime} \Phi_{i j, m n}\left(\tau-\tau^{\prime}\right) K_{m n, k l}\left(\tau^{\prime}\right), \\
\Gamma_{i j, k l}(\tau)= & \Phi_{i j, k l}(\tau)+\sum_{m, n} \int_{0}^{\tau} d \tau^{\prime} \Phi_{i j, m n}\left(\tau-\tau^{\prime}\right) \\
& \times\left(\frac{i}{\hbar}\left[\mathcal{L}_{s}\right]_{m n, k l}+\Gamma_{m n, k l}\left(\tau^{\prime}\right)\right)
\end{aligned}
$$

Closer inspection reveals that the matrix elements $\Phi_{i j, k l}(\tau)$, $\dot{\Phi}_{i j, k l}(\tau)$, and $\Xi_{i j}(t)$ can all be given in terms of overall system two-time correlation functions of the form $\operatorname{Tr}\left(\hat{A} e^{i \hat{H} t / \hbar} \hat{B} e^{-i \hat{H} t / \hbar}\right)$ as follows:

$$
\begin{aligned}
& \Phi_{i j, k l}(\tau)=\frac{i}{\hbar} \operatorname{Tr}\left\{\hat{R}|k\rangle\langle l| e^{i \hat{H} \tau / \hbar}\left[|j\rangle\langle i|, \hat{H}_{b s}\right] e^{-i \hat{H} \tau / \hbar}\right\}, \\
& \dot{\Phi}_{i j, k l}(\tau)=-\frac{1}{\hbar^{2}} \operatorname{Tr}\left\{\hat{R}|k\rangle\langle l| e^{i \hat{H} \tau / \hbar} \frac{i}{\hbar}\left[\hat{H},\left[|j\rangle\langle i|, \hat{H}_{b s}\right]\right] e^{-i \hat{H} \tau / \hbar}\right\},
\end{aligned}
$$

$$
\Xi_{i j}(t)=-\frac{i}{\hbar} \operatorname{Tr}\left\{\hat{\rho}(0) e^{i \hat{H} t / \hbar}\left[|j\rangle\langle i|, \hat{H}_{b s}\right] e^{-i \hat{H} t / \hbar}\right\} .
$$

In this context, it is also interesting to note that the BlochRedfield quantum master equation also requires two-time correlation functions as input, albeit of the free bath rather than the overall system. However, except for the rather specialized case of the harmonic bath, the computational effort involved in calculating overall system correlation functions would be comparable to that involved in calculating the freebath correlation functions. Thus, going beyond the weak coupling limit within the approach proposed here does not appear to carry a significant computational overhead.

\section{A TWO-STATE SYSTEM DIAGONALLY COUPLED TO A BATH}

The theoretical framework presented in Sec. III is very general and can be applied to practically any system, as long as one can calculate the projection-free input accurately enough and solve the corresponding GQME. In this section, we demonstrate and test the consistency of this general theoretical framework in the special case of a two-state system and a system-bath coupling which is diagonal in terms of the system's stationary state representation. Models of this type have been extensively studied in the past, for example, in the context of dephasing processes and optical spectroscopy in condensed phase systems. ${ }^{50-52}$

The overall Hamiltonian that we consider has the form of Eq. (1), with

$$
\hat{H}_{s}=\hbar \omega_{0}|e\rangle\langle e|
$$

and 


$$
\hat{H}_{b s}=\hat{U} \otimes|e\rangle\langle e| .
$$

Here, $|g\rangle$ and $|e\rangle$ are the system stationary states, such that $\hat{H}_{s}|g\rangle=0$ and $\hat{H}_{s}|e\rangle=\hbar \omega_{0}|e\rangle\left(\hbar \omega_{0}>0\right)$. It should be noted that the system-bath coupling term $\hat{H}_{b s}$ only involves the diagonal system eigenprojector $|e\rangle\langle e|$, but not the offdiagonal creation and annihilation operators, $|e\rangle\langle g|$ and $|g\rangle\langle e|$. The bath operator $\hat{U}$ corresponds to the bath-induced modulation of the energy gap between the ground and excited states. The results presented in this section are valid regardless of the specific choice of $\hat{H}_{b}$ and $\hat{U}$.

For this model, one can represent the system density operator $\hat{\sigma}(t)$ by the $2 \times 2$ matrix, whose elements are $\left\{\sigma_{g g}(t), \sigma_{g e}(t), \sigma_{e g}(t), \sigma_{e e}(t)\right\}$. The initial state of the overall system can be generally written in the following form:

$$
\begin{aligned}
\hat{\rho}(0)= & \sigma_{g g}(0) \hat{\rho}_{g} \otimes|g\rangle\left\langle g\left|+\sigma_{e e}(0) \hat{\rho}_{e} \otimes\right| e\right\rangle\langle e| \\
& +\sigma_{g e}(0) \hat{\rho}_{b} \otimes|g\rangle\left\langle e\left|+\sigma_{e g}(0) \hat{\rho}_{b}^{\dagger} \otimes\right| e\right\rangle\langle g|,
\end{aligned}
$$

where $\sigma_{g g}(0)+\sigma_{e e}(0)=1$ and $\left\{\hat{\rho}_{b}, \hat{\rho}_{b}^{\dagger}, \hat{\rho}_{g}, \hat{\rho}_{e}\right\}$ are bath operators with unity trace. The time evolution of the system density operator can be shown to be given by

$$
\begin{aligned}
\hat{\sigma}(t) & =\operatorname{Tr}_{b}\left\{e^{-i \hat{H} t / \hbar} \hat{\rho}(0) e^{i \hat{H} t / \hbar}\right\} \\
& =\left(\begin{array}{cc}
\sigma_{g g}(0) & \sigma_{g e}(0) e^{i \omega_{0} t} J_{b}(t) \\
\sigma_{e g}(0) e^{-i \omega_{0} t} J_{b}^{*}(t) & \sigma_{e e}(0)
\end{array}\right),
\end{aligned}
$$

where

$$
J_{b}(t)=\left\langle e^{i\left(\hat{H}_{b}+\hat{U}\right) t / \hbar} e^{-i \hat{H}_{b} t / \hbar}\right\rangle_{b} \equiv\left\langle\exp _{-}\left\{\frac{i}{\hbar} \int_{0}^{t} d t^{\prime} \hat{U}\left(t^{\prime}\right)\right\}\right\rangle_{b} .
$$

Here, $\langle\hat{A}\rangle_{b}=\operatorname{Tr}_{b}\left(\hat{\rho}_{b} \hat{A}\right), \quad \hat{U}(t)=e^{i \hat{H}_{b} t / \hbar} \hat{U} e^{-i \hat{H}_{b} t / \hbar}$, and $\exp _{-}\{\cdots\}$ stands for a negatively time-ordered exponential operator:

$$
\begin{aligned}
\exp _{-}\left\{\int_{t_{0}}^{t} d t^{\prime} \hat{A}\left(t^{\prime}\right)\right\} \equiv & 1+\int_{t_{0}}^{t} d t_{1} \hat{A}\left(t_{1}\right) \\
& +\int_{t_{0}}^{t} d t_{1} \int_{t_{0}}^{t_{1}} d t_{2} \hat{A}\left(t_{2}\right) \hat{A}\left(t_{1}\right)+\ldots
\end{aligned}
$$

Our goal in this section is to investigate the GQME that governs the dynamics of $\hat{\sigma}(t)$ as given in Eq. (45). The analysis starts with the general GQME in Eq. (12). It is also convenient to choose $\hat{R}=\hat{\rho}_{b}$, such that $\mathcal{L} \mathcal{Q} \hat{\rho}(0)=0$, and therefore $\hat{I}(t)=0$. It should be noted that in this case, $\hat{I}(t)=0$, regardless of the specific choices of $\hat{\rho}_{g}$ and $\hat{\rho}_{e}$ in Eq. (44).

The memory kernel superoperator $\mathcal{K}(\tau)$ can be represented by a $4 \times 4$ matrix in terms of the systems Liouville space basis set $\{|g g\rangle\rangle,|g e\rangle\rangle,|e g\rangle\rangle,|e e\rangle\rangle\}$. The memory kernel matrix elements, $\left\{\mathcal{K}_{i j, k l}(\tau)\right\}$, can be obtained by solving Eq. (37). To this end, the matrix elements $\left\{\Phi_{i j, k l}(\tau)\right\}$ and $\left\{\left[\mathcal{L}_{s}\right]_{i j, k l}(t)\right\}$ are needed as input. These matrix elements can be calculated explicitly and are given by

$$
\left[\mathcal{L}_{s}\right]_{m n, k l}= \begin{cases}-\hbar \omega_{0} & \text { if }(m n, k l)=(g e, g e), \\ +\hbar \omega_{0} & \text { if }(m n, k l)=(e g, e g), \\ 0 & \text { otherwise, }\end{cases}
$$

and

$$
\Phi_{i j, m n}(\tau)= \begin{cases}-e^{i \omega_{0} \tau} \dot{J}_{b}(\tau) & \text { if }(m n, k l)=(g e, g e), \\ -e^{-i \omega_{0} \tau} \dot{J}_{b}^{*}(\tau) & \text { if }(m n, k l)=(e g, e g), \\ 0 & \text { otherwise. }\end{cases}
$$

Substituting Eqs. (48) and (49) in Eq. (37), one can verify that all the matrix elements $\mathcal{K}_{i j, k l}(t)$, except for $\mathcal{K}_{\text {ge,ge }}(t)$ and $\mathcal{K}_{\text {eg,eg }}(t)$, satisfy a homogeneous Volterra equation of the second kind, and therefore vanish. ${ }^{49}$ This immediately verifies that $d \sigma_{g g} / d t=d \sigma_{e e} / d t=0$ [cf. Eq. (45)]. At the same time, the time evolution of $\sigma_{g e}(t)$ is seen to be dictated by the following equation:

$$
\frac{d}{d t} \sigma_{g e}(t)=i \omega_{0} \sigma_{g e}(t)-\int_{0}^{t} d \tau K(\tau) \sigma_{g e}(t-\tau),
$$

where the memory kernel function $K(\tau) \equiv \mathcal{K}_{g e, g e}(\tau)$ satisfies the following inhomogeneous Volterra equation of the second kind: ${ }^{49}$

$$
K(\tau)=-e^{i \omega_{0} \tau} \ddot{J}_{b}(\tau)-\int_{0}^{\tau} d \tau^{\prime} e^{i \omega_{0}\left(\tau-\tau^{\prime}\right)} \dot{J}_{b}\left(\tau-\tau^{\prime}\right) K\left(\tau^{\prime}\right) .
$$

Equation (50) is written in terms of the Schrödinger picture. However, working instead in terms of the interaction picture leads to even simpler equations. The transformation between the Schrödinger-picture representation of the matrix element $\sigma_{g e}(t)$ and its interaction-picture counterpart, denoted by $\widetilde{\sigma}_{g e}(t)$, is given by

$$
\widetilde{\sigma}_{g e}(t)=e^{-i \omega_{0} t} \sigma_{g e}(t) .
$$

The equation of motion for $\widetilde{\sigma}_{g e}(t)$ can be easily derived from Eq. (50), and is given by

$$
\frac{d}{d t} \widetilde{\sigma}_{g e}(t)=-\int_{0}^{t} d \tau \widetilde{K}(\tau) \widetilde{\sigma}_{g e}(t-\tau),
$$

where

$$
\widetilde{K}(\tau) \equiv K(\tau) e^{-i \omega_{0} \tau} .
$$

Finally, one can use Eq. (51) in order to derive the following inhomogeneous Volterra equation of the second kind for $\widetilde{K}(\tau)$ :

$$
\tilde{K}(\tau)=-\ddot{J}_{b}(\tau)-\int_{0}^{\tau} d \tau^{\prime} \dot{J}_{b}\left(\tau-\tau^{\prime}\right) \tilde{K}\left(\tau^{\prime}\right) .
$$

It is interesting to note that Eq. (55) can also be derived via an alternative route, starting from Eq. (45) (cf. Appendix C).

Next, we consider the limit of weak system-bath coupling. In this limit, Eq. (53) reduces to 


$$
\frac{d}{d t} \widetilde{\sigma}_{g e}(t)=-\int_{0}^{t} d \tau \widetilde{K}_{\mathrm{wc}}(\tau) \widetilde{\sigma}_{g e}(t-\tau)
$$

where

$$
\begin{aligned}
\tilde{K}_{\mathrm{wc}}(\tau) & =\frac{1}{\hbar^{2}} \operatorname{Tr}_{b}\left(\mathcal{L}_{b s} e^{-i\left(\mathcal{L}_{s}+\mathcal{L}_{b}\right) \tau / \hbar} \mathcal{L}_{b s} \hat{\rho}_{b}^{\mathrm{eq}}\right) \\
& =\frac{1}{\hbar^{2}}\langle\hat{U} \hat{U}(\tau)\rangle_{b} \equiv \frac{1}{\hbar^{2}} C_{U U}(\tau)
\end{aligned}
$$

It should also be noted that in this limit, the expansion of $J_{b}(\tau)$ in powers of the system-bath coupling can be truncated after the second order term:

$$
\begin{aligned}
J_{b}(\tau) & \approx J_{b}^{\mathrm{wc}}(\tau)=1-\frac{1}{\hbar^{2}} \int_{0}^{\tau} d \tau^{\prime} \int_{0}^{\tau^{\prime}} d \tau^{\prime \prime} C_{U U}\left(\tau^{\prime \prime}\right) \\
& =1-\int_{0}^{\tau} d \tau^{\prime} \int_{0}^{\tau^{\prime}} d \tau^{\prime \prime} \widetilde{K}_{\mathrm{wc}}\left(\tau^{\prime \prime}\right)
\end{aligned}
$$

such that

$$
\dot{J}_{b}(\tau) \approx \dot{J}_{b}^{\mathrm{wc}}(\tau)=-\frac{1}{\hbar^{2}} \int_{0}^{\tau} d \tau^{\prime} C_{U U}\left(\tau^{\prime}\right)=-\int_{0}^{\tau} d \tau^{\prime} \tilde{K}_{\mathrm{wc}}\left(\tau^{\prime}\right)
$$

and

$$
\ddot{J}_{b}(\tau) \approx \ddot{J}_{b}^{\mathrm{wc}}(\tau)=-\frac{1}{\hbar^{2}} C_{U U}(\tau)=-\widetilde{K}_{\mathrm{wc}}(\tau)
$$

It is interesting to note that $\widetilde{K}_{\mathrm{wc}}(\tau)$ coincides with $-\ddot{J}_{b}(\tau)$ at the weak coupling limit. It is also interesting to note that $\widetilde{K}(0)=-\ddot{J}_{b}(0)$ [cf. Eq. (55)], such that the initial values of $\tilde{K}(\tau)$ and $\widetilde{K}_{\text {wc }}(\tau)$ coincide in general.

Equation (56) can be further simplified if $\widetilde{K}_{\mathrm{wc}}(\tau)$ decays much faster than $\widetilde{\sigma}_{g e}(t)$. In such cases, one may replace $\widetilde{\sigma}_{g e}(t-\tau)$ in Eq. (56) by $\widetilde{\sigma}_{g e}(t)$, such that

$$
\frac{d}{d t} \widetilde{\sigma}_{g e}(t)=-\gamma(t) \widetilde{\sigma}_{g e}(t)
$$

where

$$
\gamma(t)=\int_{0}^{t} d \tau \widetilde{K}_{\mathrm{wc}}(\tau)
$$

At times longer than the lifetime of $\widetilde{K}_{\mathrm{wc}}(\tau), \gamma(t)$ becomes time independent such that

$$
\frac{d}{d t} \widetilde{\sigma}_{g e}(t)=-\gamma(\infty) \widetilde{\sigma}_{g e}(t) \equiv-\left(\frac{1}{T_{2}}+i \Delta\right) \widetilde{\sigma}_{g e}(t),
$$

where

$$
\begin{aligned}
\frac{1}{T_{2}} & \equiv \operatorname{Re}[\gamma(\infty)]=\frac{1}{\hbar^{2}} \int_{0}^{\infty} d \tau \operatorname{Re}\left[C_{U U}(\tau)\right] \\
& =\frac{1}{2 \hbar^{2}} \int_{-\infty}^{\infty} d \tau C_{U U}(\tau), \\
\Delta & \equiv \operatorname{Im}[\gamma(\infty)]=\frac{1}{\hbar^{2}} \int_{0}^{\infty} d \tau \operatorname{Im}\left[C_{U U}(\tau)\right] .
\end{aligned}
$$

\section{A SPIN-BOSON EXAMPLE}

In this section, we test and further explore the results of Sec. IV in the case of a relatively simple spin-boson model. ${ }^{53-55}$ To this end, we assume that the bath consists of $N$ independent harmonic modes of mass-weighted coordinates and momenta $\left\{Q_{j}\right\}$ and $\left\{P_{j}\right\}$, respectively:

$$
\hat{H}_{b}=\sum_{j=1}^{N}\left(\frac{\hat{P}_{j}^{2}}{2}+\frac{1}{2} \omega_{j} \hat{Q}_{j}^{2}\right) .
$$

$\hat{H}_{s}$ and $\hat{H}_{b s}$ are as in Eqs. (42) and (43), respectively, and $\hat{U}$ is assumed to be linear in the bath coordinates:

$$
\hat{U}=\sum_{j=1}^{N} c_{j} \hat{Q}_{j} .
$$

We also define the bath spectral density in the following manner:

$$
\mathcal{J}(\omega)=\sum_{j=1}^{N} \frac{\left|c_{j}\right|^{2}}{2 \omega_{j}} \delta\left(\omega-\omega_{j}\right),
$$

and assume that $\hat{\rho}_{b}=\hat{\rho}_{b}^{\mathrm{eq}}$, such that $\left\langle\hat{H}_{b s}\right\rangle_{R}=\operatorname{Tr}_{b}\left(\hat{\rho}_{b}^{\mathrm{eq}} \hat{H}_{b s}\right)=0$.

For this model, the weak coupling memory kernel $K_{\mathrm{wc}}(\tau)$ and $J_{b}(\tau)$ can be obtained analytically and are given by

$$
\begin{aligned}
K_{\mathrm{wc}}(\tau)= & \frac{1}{\hbar^{2}} C_{U U}(\tau) \\
= & \frac{1}{\hbar} \int_{0}^{\infty} d \omega \mathcal{J}(\omega)\left[\operatorname{coth}\left(\frac{\beta \hbar \omega}{2}\right) \cos (\omega t)\right. \\
& +i \sin (\omega t)], \\
J_{b}(\tau)= & \exp \left\{-\frac{1}{\hbar^{2}} \int_{0}^{\tau} d \tau^{\prime} \int_{0}^{\tau^{\prime}} d \tau^{\prime \prime} C_{U U}\left(\tau^{\prime \prime}\right)\right\} \\
= & \exp \left\{-\frac{i \tau}{\hbar} \int_{0}^{\infty} d \omega \frac{\mathcal{J}(\omega)}{\omega}-\frac{1}{\hbar} \int_{0}^{\infty} d \omega \frac{\mathcal{J}(\omega)}{\omega^{2}}\right. \\
& \left.\times\left(\operatorname{coth}\left(\frac{\beta \hbar \omega}{2}\right)(1-\cos [\omega \tau])-i \sin [\omega \tau]\right)\right\} .
\end{aligned}
$$

The fact that $K_{\mathrm{wc}}(\tau)$ and $J_{b}(t)$ can be expressed in terms of the very same two-time correlation function, $C_{U U}(\tau)$ $=\operatorname{Tr}_{b}\left[\hat{\rho}_{b}^{\mathrm{eq}} \hat{U} e^{i \hat{H}_{b} \tau / \hbar} \hat{U} e^{-i \hat{H}_{b} \tau / \hbar}\right]$, should be attributed to the fact 
that the bath is harmonic, such that a second order cumulant expansion of $J_{b}(t)$ is exact. It should be noted that a Gaussian approximation can also be employed for calculating $J_{b}(t)$ in the case of an anharmonic bath, by truncating the corresponding cumulant expansion at second order.

Finally, we note that although the Markovian weak coupling quantum master equation [Eq. (61)] is, in principle, more approximate than the non-Markovian weak coupling quantum master equation [Eq. (56)], it actually yields the exact result for this spin-boson model. This is a fortuitous coincidence, which is intimately related to the fact that the bath is harmonic. More specifically, $d \widetilde{\sigma}_{g e} / d t=-\gamma(t) \widetilde{\sigma}_{g e}(t) \mathrm{im}-$ plies that [cf. Eqs. (62), (57), and (45)]

$$
\begin{aligned}
\frac{\widetilde{\sigma}_{g e}(t)}{\widetilde{\sigma}_{g e}(0)} & =\exp \left(-\int_{0}^{t} d \tau \gamma(\tau)\right) \\
& =\exp \left\{-\int_{0}^{t} d \tau \int_{0}^{\tau} d \tau^{\prime} K_{\mathrm{wc}}\left(\tau^{\prime}\right)\right\} \\
& =\exp \left\{-\frac{1}{\hbar^{2}} \int_{0}^{t} d \tau \int_{0}^{\tau} d \tau^{\prime} C_{U U}\left(\tau^{\prime}\right)\right\}=J_{b}(t) .
\end{aligned}
$$

The calculation reported below were performed for Ohmic and Debye spectral densities with exponential cutoffs:

$$
\begin{aligned}
& \mathcal{J}_{O}(\omega)=\left\{\begin{array}{cl}
\kappa_{o} \gamma^{2} \omega e^{-\gamma \omega} & \text { for } \omega \geqslant 0, \\
0 & \text { for } \omega<0,
\end{array}\right. \\
& \mathcal{J}_{D}(\omega)=\left\{\begin{array}{cc}
\kappa_{d}\left(\gamma^{4} / 6\right) \omega^{3} e^{-\gamma \omega} & \text { for } \omega \geqslant 0, \\
0 & \text { for } \omega<0 .
\end{array}\right.
\end{aligned}
$$

In both cases, the parameters $\beta$ and $\gamma$ were assigned as $\beta$ $=1.0$ and $\gamma=5.0$, respectively.

\section{A. Ohmic bath}

We first consider the case of an Ohmic bath [cf. Eq. (71)]. The real and imaginary parts of the memory kernel $\widetilde{K}(\tau)$ were calculated by numerically solving the corresponding inhomogeneous Volterra equation of the second kind, Eq. $(55){ }^{56}$ The real and imaginary parts of the resulting memory kernels are shown in Fig. 1 for values of $\kappa_{o}$ ranging from 0.0001, which corresponds to weak system-bath coupling, to 0.1000 , which corresponds to strong system-bath coupling. Also shown on this plot is the weak coupling memory kernel, $K_{\mathrm{wc}}(\tau)$ [cf. Eq. (68)]. The memory kernels all converge to the same value at $t=0$, as expected. Furthermore, the exact $\widetilde{K}(\tau)$ converges to its weak coupling counterpart, $\widetilde{K}_{\text {wc }}(\tau)$, at the limit of weak system-bath coupling. However, increasingly larger deviations are observed between $\widetilde{K}(\tau)$ and $\widetilde{K}_{\text {wc }}(\tau)$ as the coupling becomes stronger. More specifically, $\widetilde{K}(\tau)$ becomes shorter-lived as $\kappa_{o}$ increases, and develops a region where $\tilde{K}(\tau)$ becomes increasingly more negative.

The relaxation of the real and imaginary parts of $\widetilde{\sigma}_{g e}(t)$ in the case of strong system-bath coupling $\left(\kappa_{o}=0.1000\right)$ is shown in Fig. 2. The results obtained by numerically solving the GQME with the exact memory kernel, Eq. (53), via the

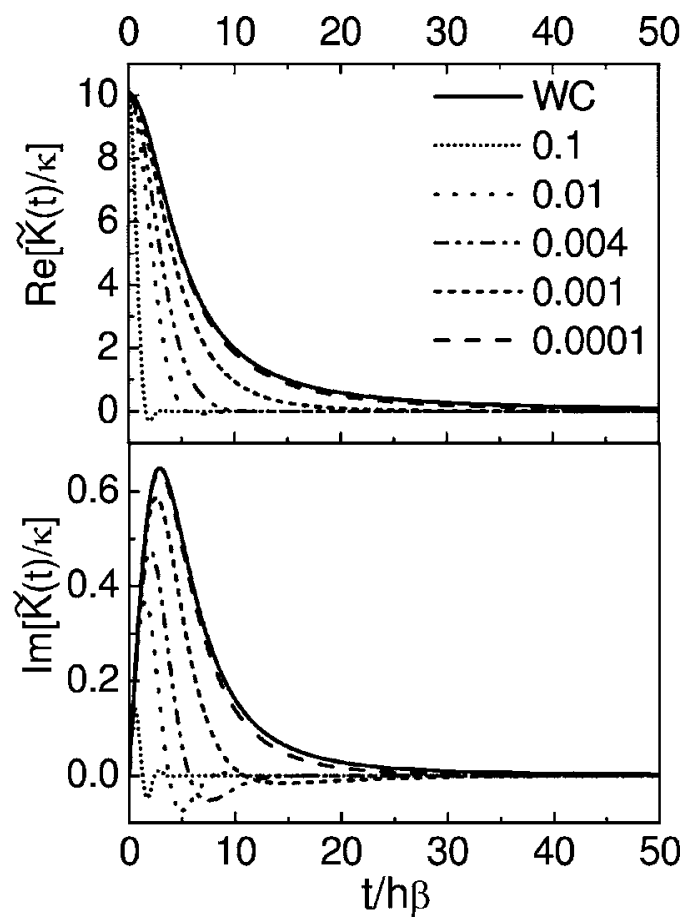

FIG. 1. The memory kernel in the case of an Ohmic bath $(\beta=1.0, \gamma=5.0)$. Shown are the real and imaginary parts of the exact memory kernel, $\tilde{K}(\tau)$, for the indicated values of $\kappa_{o}$. Also shown for comparison is the corresponding weak coupling approximation, $\widetilde{K}_{\mathrm{wc}}(\tau)$.

fourth order Runge-Kutta method ${ }^{56}$ are seen to coincide with the exact result. It should also be noted that the system relaxation occurs on a time scale which is comparable to the lifetime of the exact memory kernel. Finally, the prediction

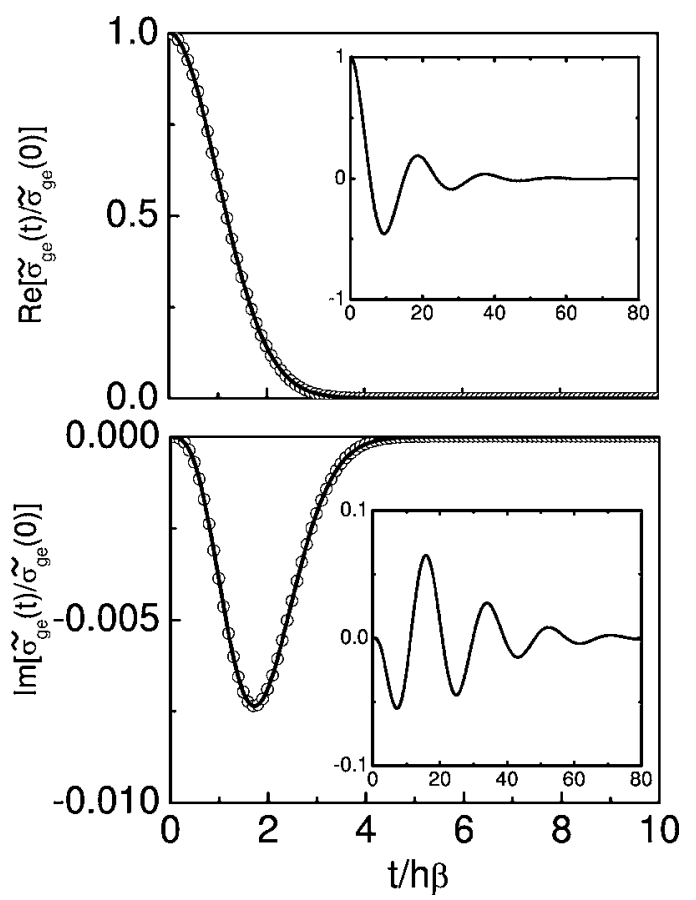

FIG. 2. The real and imaginary parts of $\widetilde{\sigma}_{g e}(t)$ as it relaxes to equilibrium in the case of an Ohmic bath $\left(\kappa_{o}=0.1000, \beta=1.0, \gamma=5.0\right)$. Shown are the exact results, Eq. (45) (solid line), and the results obtained by solving the GQME (circles). The inset shows the corresponding predictions based on the weak system-bath coupling treatment [cf. Eq. (56)]. 


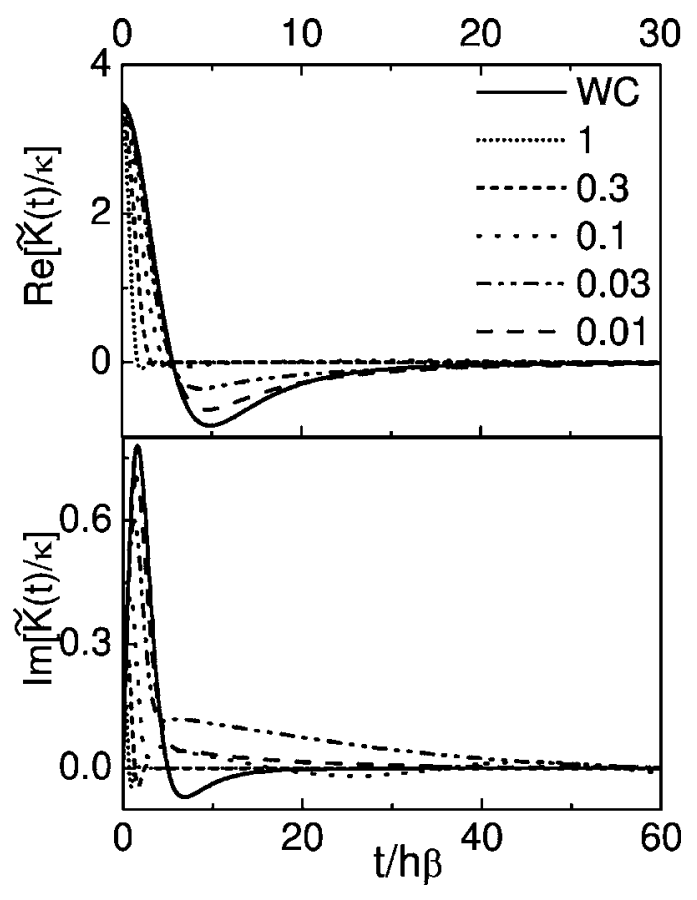

FIG. 3. The memory kernel in the case of a Debye bath $(\beta=1.0, \gamma=5.0)$. Shown are the real and imaginary parts of the exact memory kernel, $\widetilde{K}(\tau)$, for the indicated values of $\kappa_{d}$. Also shown for comparison is the corresponding weak coupling approximation, $\widetilde{K}_{\text {wc }}(\tau)$.

of the weak coupling treatment, Eq. (56), is clearly underdamped, longer lived, and qualitatively incorrect (cf. inset of Fig. 2).

\section{B. Debye bath}

We next consider the Debye bath case [cf. Eq. (72)]. The real and imaginary parts of the memory kernels are shown in Fig. 3 for values of $\kappa_{d}$ ranging from 0.01 (weak system-bath coupling) to 1.000 (strong system-bath coupling). As in the case of the Ohmic bath, $\widetilde{K}(\tau)$ becomes increasingly shorterlived in comparison to $\widetilde{K}(\tau)$ as the coupling becomes stronger. It should also be noted that the detailed behavior of $\tilde{K}(\tau)$ is different in comparison to the Ohmic bath.

The relaxation of the real and imaginary parts of $\tilde{\sigma}_{g e}(t)$ in the case of strong system-bath coupling $\left(\kappa_{d}=1.00\right)$ is shown in Fig. 4. The results obtained by numerically solving the GQME with the exact memory kernel are seen to coincide with the exact result. At the same time, the prediction of the weak coupling treatment, Eq. (56), is once again clearly underdamped, longer lived, and qualitatively incorrect (cf. inset of Fig. 4).

\section{SUMMARY}

In this paper, we proposed a new approach for calculating the memory kernel and inhomogeneous term of the GQME, for arbitrary initial state and system-bath coupling. The proposed theoretical framework allows for the calculation of the memory kernel and inhomogeneous term by solving an inhomogeneous Volterra equation of the second kind for each. The new approach is considerably more general and

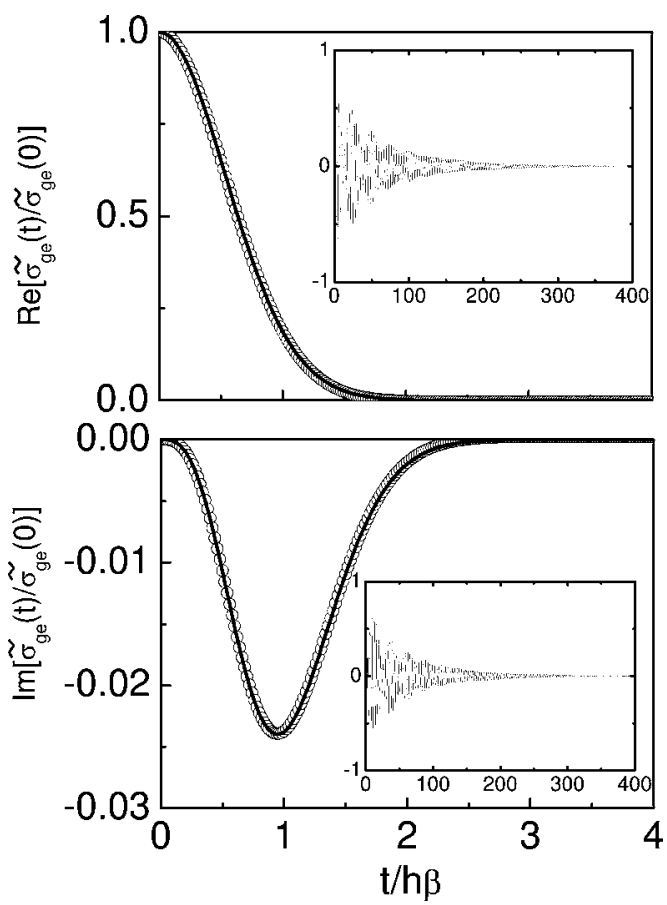

FIG. 4. The real and imaginary parts of $\widetilde{\sigma}_{g e}(t)$ as it relaxes to equilibrium in the case of a Debye bath $\left(\kappa_{d}=1.000, \beta=1.0, \gamma=5.0\right)$. Shown are the exact results, Eq. (45) (solid line), and the results obtained by solving the GQME (circles). The inset shows the corresponding predictions based on the weak system-bath coupling treatment [cf. Eq. (56)].

streamlined in comparison to our original proposal. ${ }^{16}$ It can accommodate a wide range of possible projection operators and can even deal with explicitly time-dependent Hamiltonians. The application of the new approach to the case of a two-state system with diagonal system-bath coupling was considered, and its self-consistency was demonstrated on a spin-boson model.

The next natural step is to apply the new method to systems where the exact quantum dynamics is not known. Of particular interest are systems where the bath is anharmonic (e.g., liquid solutions), systems that involve population transfer between the system quantum states (either nonradiative or laser driven), and systems where initial system-bath correlations are important. It should be noted that in most cases of practical interest, calculating the quantum-mechanical projection-free two-time correlation functions in Eqs. (39)-(41) will have to be done numerically. Furthermore, approximate semiclassical and mixed quantum-classical techniques, many of which were especially designed for computing such two-time correlation functions, will have to be employed in applications to anharmonic systems such as liquid solutions (e.g., see Refs. 39 and 40). In such cases, the quality of the results will obviously depend on the reliability of the underlying approximations. However, it should be noted that approximating the memory kernel, followed by solving the GQME, would often lead to more accurate system dynamics than that obtained by direct application of the same approximation. ${ }^{39,40}$ Work on these issues is underway, and will be reported in forthcoming publications. 


\section{ACKNOWLEDGMENT}

This project was supported by the National Science Foundation FOCUS Center, through Grant No. PHY0114336.

\section{APPENDIX A: EVALUATION OF THE MEMORY KERNEL AND INHOMOGENEOUS TERM FOR A SYSTEM WITH A TIME-DEPENDENT HAMILTONIAN}

In this appendix, we generalize the formalism described in Secs. II and III to cases where $\hat{H}$ is explicitly time dependent. We focus on the rather prevalent situation where the system is driven by an external time-dependent classical field. In such cases, one can assume that only $\hat{H}_{s}$ is explicitly time dependent. A commonly encountered example corresponds to photoexcitation by laser light of a chromophore embedded in a condensed phase host.

\section{The GQME for a system with an explicitly time-dependent Hamiltonian}

Consider an overall system with the following general quantum-mechanical Hamiltonian:

$$
\hat{H}=\hat{H}_{s}(t)+\hat{H}_{b}+\hat{H}_{b s}
$$

where the system Hamiltonian, $\hat{H}_{s}(t)$, is now assumed to be explicitly time dependent, while $\hat{H}_{b}$ and $\hat{H}_{b s}$ are as in Sec. II. Following a similar procedure to that outlined in Sec. II, it can be shown that the GQME in this case is given by ${ }^{6}$

$$
\frac{d}{d t} \hat{\sigma}(t)=-\frac{i}{\hbar} \mathcal{L}_{s}(t) \hat{\sigma}(t)-\int_{0}^{t} d \tau \mathcal{K}(\tau, t) \hat{\sigma}(t-\tau)+\hat{I}(t) .
$$

A detailed derivation of the explicit expressions for the memory kernel, $\mathcal{K}(\tau, t)$, and inhomogeneous term, $\hat{I}(t)$, can be found in Ref. 6 . The final results are given by

$$
\begin{aligned}
\mathcal{K}(\tau, t)= & \frac{1}{\hbar^{2}} \operatorname{Tr}_{b}\left\{\mathcal{L}_{b s} \exp _{+}\left\{-\frac{i}{\hbar} \int_{t-\tau}^{t} d t^{\prime} \mathcal{Q} \mathcal{L}\left(t^{\prime}\right)\right\}\right. \\
& \times \mathcal{Q L}(t-\tau) \hat{R}\}, \\
\hat{I}(t)=- & \frac{i}{\hbar} \operatorname{Tr}_{b}\left\{\mathcal{L}_{b s} \exp _{+}\left\{-\frac{i}{\hbar} \int_{0}^{t} d t^{\prime} \mathcal{Q} \mathcal{L}\left(t^{\prime}\right)\right\} \mathcal{Q} \hat{\rho}(0)\right\},
\end{aligned}
$$

where $\exp _{+}\{\ldots\}$ stands for a positively time-ordered exponential superoperator:

$$
\begin{aligned}
\exp _{+}\left\{\int_{t_{0}}^{t} d t^{\prime} \mathcal{A}\left(t^{\prime}\right)\right\} \equiv & 1+\int_{t_{0}}^{t} d t_{1} \mathcal{A}\left(t_{1}\right) \\
& +\int_{t_{0}}^{t} d t_{1} \int_{t_{0}}^{t_{1}} d t_{2} \mathcal{A}\left(t_{1}\right) \mathcal{A}\left(t_{2}\right)+\ldots
\end{aligned}
$$

It should be noted that the memory kernel is now explicitly $t$ dependent (as well as $\tau$ dependent).

\section{Evaluation of the memory kernel and inhomogeneous term}

The identity in Eq. (17) can be generalized to the case of an explicitly time-dependent Hamiltonian:

$$
\begin{aligned}
\exp _{+}\left\{-\frac{i}{\hbar} \int_{t_{0}}^{t} d t^{\prime} \mathcal{Q} \mathcal{L}\left(t^{\prime}\right)\right\} \\
=\exp _{+}\left\{-\frac{i}{\hbar} \int_{t_{0}}^{t} d t^{\prime} \mathcal{L}\left(t^{\prime}\right)\right\}+\frac{i}{\hbar} \int_{t_{0}}^{t} d t^{\prime} \\
\times \exp _{+}\left\{-\frac{i}{\hbar} \int_{t^{\prime}}^{t} d t^{\prime \prime} \mathcal{L}\left(t^{\prime \prime}\right)\right\} \mathcal{P} \mathcal{L}\left(t^{\prime}\right) \\
\times \exp _{+}\left\{-\frac{i}{\hbar} \int_{t_{0}}^{t^{\prime}} d t^{\prime \prime} \mathcal{Q L}\left(t^{\prime \prime}\right)\right\} .
\end{aligned}
$$

Equation (A6) can be derived by integrating both sides of the differential equation

$$
\begin{aligned}
\frac{d}{d t}\left[\exp _{-}\left\{\frac{i}{\hbar} \int_{t_{0}}^{t} d t^{\prime} \mathcal{L}\left(t^{\prime}\right)\right\} \exp _{+}\left\{-\frac{i}{\hbar} \int_{t_{0}}^{t} d t^{\prime} \mathcal{Q L}\left(t^{\prime}\right)\right\}\right] \\
=\exp _{-}\left\{\frac{i}{\hbar} \int_{t_{0}}^{t} d t^{\prime} \mathcal{L}\left(t^{\prime}\right)\right\} \mathcal{P} \mathcal{L}(t) \\
\quad \times \exp _{+}\left\{-\frac{i}{\hbar} \int_{t_{0}}^{t} d t^{\prime} \mathcal{Q} \mathcal{L}\left(t^{\prime}\right)\right\}
\end{aligned}
$$

from $t_{0}$ to $t$, and multiplying the result from the left by $\exp _{+}\left\{-(i / \hbar) \int_{t_{0}}^{t} d t^{\prime} \mathcal{L}\left(t^{\prime}\right)\right\}$. Here, $\exp _{-}\{\ldots\}$ stands for a negatively time-ordered exponential superoperator:

$$
\begin{aligned}
\exp _{-}\left\{\int_{t_{0}}^{t} d t^{\prime} \mathcal{A}\left(t^{\prime}\right)\right\} \equiv & 1+\int_{t_{0}}^{t} d t_{1} \mathcal{A}\left(t_{1}\right) \\
& +\int_{t_{0}}^{t} d t_{1} \int_{t_{0}}^{t_{1}} d t_{2} \mathcal{A}\left(t_{2}\right) \mathcal{A}\left(t_{1}\right)+\ldots
\end{aligned}
$$

Substituting Eq. (A6) in Eq. (A4), and using the projection operator in Eq. (11) then leads to an equation which is analogous to Eq. (19):

$$
\hat{I}(t)=\Xi(t)+\Phi(0, t) \hat{\sigma}(0)+\int_{0}^{t} d \tau \Phi(\tau, t) \hat{I}(\tau),
$$

where

$$
\Xi(t)=-\frac{i}{\hbar} \operatorname{Tr}_{b}\left[\mathcal{L}_{b s} \exp _{+}\left\{-\frac{i}{\hbar} \int_{0}^{t} d t^{\prime} \mathcal{L}\left(t^{\prime}\right)\right\} \hat{\rho}(0)\right]
$$

and

$$
\Phi(\tau, t)=\frac{i}{\hbar} \operatorname{Tr}_{b}\left\{\mathcal{L}_{b s} \exp _{+}\left\{-\frac{i}{\hbar} \int_{\tau}^{t} d t^{\prime} \mathcal{L}\left(t^{\prime}\right)\right\} \hat{R}\right\} .
$$

Similarly, substituting Eq. (A6) in Eq. (A3), and using the projection operator in Eq. (11), leads to the following equation for the memory kernel, which is analogous to Eq. (22): 


$$
\begin{aligned}
\mathcal{K}(\tau, t)= & \frac{\partial}{\partial \tau} \Phi(\tau, t)+\frac{i}{\hbar} \Phi(\tau, t) \mathcal{L}_{s}(t) \\
& +\int_{0}^{\tau} d \tau^{\prime} \Phi\left(\tau-\tau^{\prime}, t\right) \mathcal{K}\left(\tau^{\prime}, t-\tau\right)
\end{aligned}
$$

\section{APPENDIX B: EVALUATION OF THE MEMORY KERNEL AND INHOMOGENEOUS TERM IN THE CASE OF A SYMMETRIZED PROJECTION OPERATOR}

In this appendix, we generalize the formalism described in Secs. II and III to the case of a symmetrized projector operator of the form

$$
\mathcal{P}(\cdot)=\frac{1}{2}\left[\hat{R} \operatorname{Tr}_{b}(\cdot)+\operatorname{Tr}_{b}(\cdot) \hat{R}^{\dagger}\right]
$$

where $\hat{R}$ is an overall system operator, $\hat{R}^{\dagger}$ is its Hermitian conjugate, and $\operatorname{Tr}_{b}(\hat{R})=\operatorname{Tr}_{b}\left(\hat{R}^{\dagger}\right)=\hat{1}_{s}$. For example, RomeroRochin et al. ${ }^{13-15}$ have employed a symmetrized projector operator of this form, with

$$
\hat{R}=e^{-\beta \hat{H}}\left[\operatorname{Tr}_{b}\left(e^{-\beta \hat{H}}\right)\right]^{-1},
$$

in order to retain the Hermiticity of the individual terms in the GQME and eliminate the inhomogeneous term in cases where $\hat{\rho}(0)=e^{-\beta \hat{H}} / \operatorname{Tr}\left(e^{-\beta \hat{H}}\right)$. that

Similar to Eq. (2), we assume without loss of generality

$$
\left\langle\hat{H}_{b s}\right\rangle_{R} \equiv \frac{1}{2}\left[\operatorname{Tr}_{b}\left(\hat{R} \hat{H}_{b s}\right)+\operatorname{Tr}_{b}\left(\hat{H}_{b s} \hat{R}^{\dagger}\right)\right]=0 .
$$

The GQME in matrix form can then be shown to have the same form as Eq. (35), with

$$
\mathcal{K}_{i j, k l}(\tau)=\operatorname{Tr}\left\{(|i\rangle\langle j|)^{\dagger} \mathcal{L}_{b s} e^{-i \mathcal{Q} \mathcal{L} \tau / \hbar} \mathcal{Q} \mathcal{L}\left(\hat{R}|k\rangle\langle l|+| k\rangle\langle l| \hat{R}^{\dagger}\right)\right\}
$$

and

$$
\hat{I}_{i j}(t)=\frac{i}{\hbar}\left\langle i\left|\mathcal{L}_{b s} e^{-i \mathcal{Q} \mathcal{L} t / \hbar}\left[\hat{\rho}(0)-\frac{1}{2}\left(\hat{R} \hat{\sigma}(0)+\hat{\sigma}(0) \hat{R}^{\dagger}\right)\right]\right| j\right\rangle .
$$

As in the case of the one-sided projector operator, the evaluation of the matrices $\left\{\mathcal{K}_{i j, k l}(\tau)\right\}$ and $\left\{\hat{I}_{i j}(t)\right\}$ is facilitated by the identity in Eq. (17). The matrix $\left\{\hat{I}_{i j}(t)\right\}$ can be shown to satisfy an equation of the same form as Eq. (36), where $\Xi_{i j}(t)$ is as in Eq. (41), and

$$
\begin{aligned}
\Phi_{i j, k l}(t)= & \frac{i}{\hbar} \operatorname{Tr}\left\{\frac{1}{2}\left[\hat{R}|k\rangle\langle l|+| k\rangle\langle l| \hat{R}^{\dagger}\right] e^{i \hat{H} t / \hbar}[|j\rangle\right. \\
& \left.\left.\times\langle i|, \hat{H}_{b s}\right] e^{-i \hat{H} t / \hbar}\right\}
\end{aligned}
$$

is the symmetrized analog of Eq. (39). The matrix $\left\{\mathcal{K}_{i j, k l}(\tau)\right\}$ can be shown to satisfy an equation of the same form as Eq. (37), where $\Phi_{i j, k l}(t)$ is as in Eq. (B6).

\section{APPENDIX C: AN ALTERNATIVE DERIVATION OF EQ. (55)}

According to Eq. (45)

$$
\frac{\widetilde{\sigma}_{g e}(t)}{\tilde{\sigma}_{g e}(0)}=J_{b}(t)
$$

Taking the time derivative of Eq. (C1), and employing the ansatz $d \widetilde{\sigma}_{g e} / d t=-\int_{0}^{t} d \tau \tilde{K}(\tau) \widetilde{\sigma}_{g e}(t-\tau)$, then leads to the following equation:

$$
\dot{J}_{b}(t)=-\int_{0}^{t} d \tau \tilde{K}(\tau) J_{b}(t-\tau) .
$$

Taking the derivative with respect to $t$ of both sides of Eq. (C2) and rearranging, then leads to Eq. (55).

${ }^{1}$ S. Nakajima, Prog. Theor. Phys. 20, 948 (1958).

${ }^{2}$ R. Zwanzig, Lect. Theor. Phys. 3, 106 (1960).

${ }^{3}$ R. Zwanzig, J. Chem. Phys. 33, 1338 (1960).

${ }^{4}$ R. Zwanzig, Physica (Utrecht) 30, 1109 (1964).

${ }^{5}$ I. Prigogine and P. Resibois, Physica (Amsterdam) 27, 629 (1961).

${ }^{6}$ F. Haake, Springer Tracts Mod. Phys. 66, 98 (1973).

${ }^{7}$ H. Grabert, Projection Operator Techniques in Nonequilibrium Statistical Mechanics (Springer-Verlag, Berlin, 1982).

${ }^{8}$ R. Alicki and K. Lendi, Quantum Dynamical Semigroups and Applications (Springer-Verlag, Berlin, 1987).

${ }^{9}$ V. May and O. Kühn, Charge and Energy Transfer Dynamics in Molecular Systems (Wiley-VCH, Berlin, 2000).

${ }^{10}$ B. Yoon, J. M. Deutch, and J. H. Freed, J. Chem. Phys. 62, 4687 (1975).

${ }^{11}$ I. Oppenheim, K. E. Shuler, and G. H. Weiss, Stochastic Processes in Chemical Physics: The Master Equation (MIT, Cambridge, MA, 1977).

${ }^{12}$ S. Mukamel, I. Oppenheim, and J. Ross, Phys. Rev. A 17, 1988 (1978).

${ }^{13}$ V. Romero-Rochin and I. Oppenheim, J. Stat. Phys. 53, 307 (1988).

${ }^{14}$ V. Romero-Rochin and I. Oppenheim, Physica A 155, 52 (1989).

${ }^{15}$ V. Romero-Rochin, A. Orsky, and I. Oppenheim, Physica A 156, 244 (1989).

${ }^{16}$ Q. Shi and E. Geva, J. Chem. Phys. 119, 12063 (2003).

${ }^{17}$ K. Blum, Density Matrix Theory and Applications (Plenum, New York, 1996).

${ }^{18}$ R. K. Wangsness and F. Bloch, Phys. Rev. 89, 728 (1953).

${ }^{19}$ A. G. Redfield, IBM J. Res. Dev. 1, 19 (1957).

${ }^{20}$ N. G. van Kampen, Stochastic Processes in Physics and Chemistry (North-Holland, Amsterdam, 1981).

${ }^{21}$ R. Kubo, M. Toda, and N. Hashitsume, Statistical Physics II: Nonequilibrium Statistical Mechanics (Springer-Verlag, New York, 1985).

${ }^{22}$ B. B. Laird, J. Budimir, and J. L. Skinner, J. Chem. Phys. 94, 4391 (1991).

${ }^{23}$ W. T. Pollard and R. A. Friesner, J. Chem. Phys. 100, 5054 (1994).

${ }^{24}$ W. T. Pollard, A. K. Felts, and R. A. Friesner, Adv. Chem. Phys. 93, 77 (1996).

${ }^{25}$ E. Geva, R. Kosloff, and J. L. Skinner, J. Chem. Phys. 102, 8541 (1995).

${ }^{26}$ E. Geva and R. Kosloff, J. Chem. Phys. 104, 7681 (1996).

${ }^{27}$ D. Kohen, C. C. Marston, and D. J. Tannor, J. Chem. Phys. 107, 5236 (1997).

${ }^{28}$ J. Cao, J. Chem. Phys. 107, 3204 (1997).

${ }^{29}$ Y. J. Yan, Phys. Rev. A 58, 2721 (1998).

${ }^{30}$ M. Berman, R. Kosloff, and H. Tal-Ezer, J. Phys. A 25, 1283 (1992).

${ }^{31}$ G. Ashkenazi, G. Ashkenazi, U. Banin, A. Bartana, R. Kosloff, and S. Ruhman, Adv. Chem. Phys. 100, 229 (1997).

${ }^{32}$ G. Ashkenazi, R. Kosloff, and M. A. Ratner, J. Am. Chem. Soc. 121, 3386 (1999)

${ }^{33}$ R. Kosloff, M. A. Ratner, and W. B. Davis, J. Chem. Phys. 106, 7036 (1997).

${ }_{35}^{34}$ A. Suárez and R. Silbey, J. Chem. Phys. 94, 4809 (1991).

${ }^{35} \mathrm{D}$. Li and G. A. Voth, J. Phys. Chem. 95, 10425 (1991).

${ }^{36}$ N. Makri, J. Math. Phys. 36, 2430 (1995).

${ }^{37}$ N. Makri, J. Phys. Chem. A 102, 4414 (1998).

${ }^{38}$ N. Makri, Annu. Rev. Phys. Chem. 50, 167 (1999).

${ }^{39}$ Q. Shi and E. Geva, J. Chem. Phys. 120, 10647 (2004).

${ }^{40}$ Q. Shi and E. Geva, J. Chem. Phys. 121, 3393 (2004).

${ }^{41}$ R. Kubo, M. Toda, and N. Hashitsume, Statistical Physics II: Nonequilibrium Statistical Mechanics (Springer-Verlag, Berlin, 1983).

${ }^{42}$ J. T. Hynes and J. M. Deutch, in Physical Chemistry, edited by D. Henderson (Academic, New York, 1975), Vol. XIB.

${ }^{43}$ R. Zwanzig, Nonequilibrium Statistical Mechanics (Oxford University 
Press, New York, 2001).

${ }^{44}$ W. Heitler, Quantum Theory of Radiation (Oxford, London, 1954).

${ }^{45}$ U. Fano, Phys. Rev. 131, 259 (1963).

${ }^{46}$ A. Ben-Reuven, Phys. Rev. 141, 34 (1966).

${ }^{47}$ H. Mori, Prog. Theor. Phys. 33, 423 (1965).

${ }^{48}$ H. Grabert, Z. Phys. B 26, 79 (1977).

${ }^{49}$ P. M. Morse and H. Feshbach, Methods of Theoretical Physics (McGrawHill, New York, 1953).

${ }^{50}$ S. Mukamel, Principles of Nonlinear Optical Spectroscopy (Oxford, New York, 1995).
${ }^{51}$ M. D. Stephens, J. G. Saven, and J. L. Skinner, J. Chem. Phys. 106, 2129 (1997).

${ }^{52}$ J. L. Skinner and W. E. Moerner, J. Phys. Chem. 100, 13251 (1996).

${ }^{53}$ A. J. Leggett, A. J. Leggett, S. Chakravarty, A. T. Dorsesy, M. P. A. Fisher, A. Garg, and W. Zwerger, Rev. Mod. Phys. 59, 1 (1987).

${ }^{54}$ J. L. Skinner and D. Hsu, Adv. Chem. Phys. 65, 1 (1986).

${ }^{55}$ S. A. Egorov, E. Rabani, and B. J. Berne, J. Chem. Phys. 108, 1407 (1998).

${ }^{56}$ W. H. Press, B. P. Flannery, S. A. Teukolsky, and W. T. Vetterling, Numerical Recipes (Cambridge University Press, Cambridge, 1986). 